\title{
Optimized Wireless Sensor Node Multidimensional Routing using Fuzzy Clustering and Chaotic Gravitational Search Algorithm
}

\author{
Dr. D. Sivaganesan, \\ Professor, \\ Department of Computer Engineering, \\ PSG Institute of Technology and Applied Research, \\ Coimbatore, India. \\ Email: sivaganesan@psgitech.ac.in
}

\begin{abstract}
A network of tiny sensors located at various regions for sensing and transmitting information is termed as wireless sensor networks. The information from multiple network nodes reach the destination node or the base station where data processing is performed. In larger search spaces, the clustering mechanisms and routing solutions provided by the existing heuristic algorithms are often inefficient. The sensor node resources are depleted by un-optimized processes created by reduced routing and clustering optimization levels in large search spaces. Chaotic Gravitational Search Algorithm and Fuzzy based clustering schemes are used to overcome the limitations and challenges of the conventional routing systems. This enables effective routing and efficient clustering in large search spaces. In each cluster, among the available nodes, appropriate node is selected as the cluster head. Reduction in delay, increase in energy consumption, increase in network lifetime and improvement of the network clustering accuracy are evident from the simulation results.
\end{abstract}

Keywords: Fuzzy clustering, Chaotic Gravitational Search Algorithm, Energy efficiency, Wireless sensor nodes, Clustering accuracy

\section{Introduction}

A network of tiny sensors located at various regions for sensing and transmitting information is termed as wireless sensor networks (WSNs) [1]. The information from 
multiple network nodes reach the destination node or the base station where data processing is performed. Data collection and analysis is performed using the WSNs in several applications inclusive of disaster management, weather forecasting, military applications, security systems, agriculture and medicine. Battery, microcontroller and transceiver are the major components available in each sensor node of the WSN [2]. Optimal deployment of the sensor is essential as it is expensive to deploy several sensor at multiple locations. Congestion control, error control, flow control and routing applications are performed by the WSNs based on the network size [3]. A combination of mobile or static sink nodes with mobile or static member nodes may be used for designing such networks. For several applications, the combination that involves mobile member nodes and static sink nodes prove beneficial in several ways [4].

Other sensor nodes are used to forward the information obtained from the WSN sensor nodes directly to the destination node. A base station termed as sink is available in each sensor node [5]. A group of nodes in similar networks can cover a large area. In order to transmit the gathered information from the sensor nodes to the sink nodes, performing a one-hop or direct communication is not possible [6]. There is an inconsistency in the distances between multiple nodes. Energy may be preserved and direct communication can be performed by the nodes closer to the sink in such cases. However, nodes that are located farther requires the help of other sensor nodes for routing data packets to the required destination node. Rather than transmitting their own information, data forwarding will cause energy draining in the sensor nodes that are closer to the destination node in such scenarios [7]. A cluster head may be chosen by grouping the sensor nodes into clusters to overcome this issue and perform routing and data transmission with the help of the cluster heads. During the WSN design, routing through cluster members, maintaining the cluster, and formation of new cluster in mobility scenarios are the major design challenges that are to be addressed.

\section{Literature Survey}

Several literatures are available on energy efficient WSN routing schemes. Researchers find the optimal routes in vehicular ad hoc networks (VANETs) by applying 
biological inspirations like swarm intelligence and evolutionary routing to overcome the limitations of the existing routing protocols [8]. Prediction based mobility model, group based mobility model, mobility based geographical map and random mobility are the mobility model used in routing VANETs during vehicular movement. Selection and implementation of the appropriate mobility model is possible through this survey. Estimation of the network lifetime of residual links in mobile ad hoc networks (MANET) are performed by using various distance measurement metrics for achieving minimum distance based on the hops between the nodes [9]. The residual links lifetime in MANET can be predicted using an algorithm that analyses the change in velocity between any two nodes.

The WSN lifetime issue may be overcome with routing algorithms like Tabu search, Particle Swarm Optimization (PSO) [10], Ant Colony Optimization (ACO) [11], Genetic Algorithm (GA) [12] and simulated annealing (SA) [13] based on the meta-heuristics perspective. Operators like determination, evaluation and transition in these algorithms are determined and the characteristics of each algorithm are distinguished. Challenges regarding total live nodes, data routing and set cover are overcome with a novel algorithm. Set cover problem may be overcome with a combination of SA based GA and Tabu search algorithm. Routing issue is overcome by ACO and total live nodes issue is addressed by PSO algorithm [6]. The meta-heuristic algorithms may be implemented in an efficient manner on the basis of this work. For cluster based routing, the honey bee foraging based artificial bee colony $(\mathrm{ABC})$ algorithm is proposed as the throughput and lifetime of the network is affected by the routing process. The data gathered by the clusters can be transmitted to the base station only by the cluster heads in cluster based routing algorithms. In such scenarios, the optimization problems may be addressed by the $\mathrm{ABC}$ algorithm. The throughput, scalability, link quality, and energy efficiency may be achieved as the location unaware sensors are exploited for information and based on the signal strength, the link quality varies [14]. Here routing is performed in a distributed manner while clustering is performed through a centralized methodology. 


\section{Proposed Model}

The similarity concept of the conventional clustering mechanisms are overlooked while the attractive force between two nodes is considered for developing the proposed fuzzy based clustering scheme. When each node moves at a slow speed, a resultant force is created during which clusters are formed. Two nodes are assumed to be nearby if the force between them is large. Consider two nodes $\mathrm{N}_{\mathrm{a}}$ and $\mathrm{N}_{\mathrm{b}}$, the similarities between the nodes may be represented by $\sigma\left(\mathrm{N}_{\mathrm{a}}, \mathrm{N}_{\mathrm{b}}\right)$ and distance is given by $\mathrm{D}\left(\mathrm{N}_{\mathrm{a}}, \mathrm{N}_{\mathrm{b}}\right)$. The clusters are specified earlier to avoid wide clusters. A single center point may be modelled for this purpose by the clustering algorithm. The attraction force $\mathrm{F}_{\mathrm{ab}}$ on $\mathrm{N}_{\mathrm{a}}$ caused by $\mathrm{N}_{\mathrm{b}}$ depends on the direction of the nodes and distance between the nodes. Clusters are formed when the nodes come close to each other in the specific environment. They are assumed to be similar. Figure 1 represents the fuzzy rule based cluster head selection in every cluster.

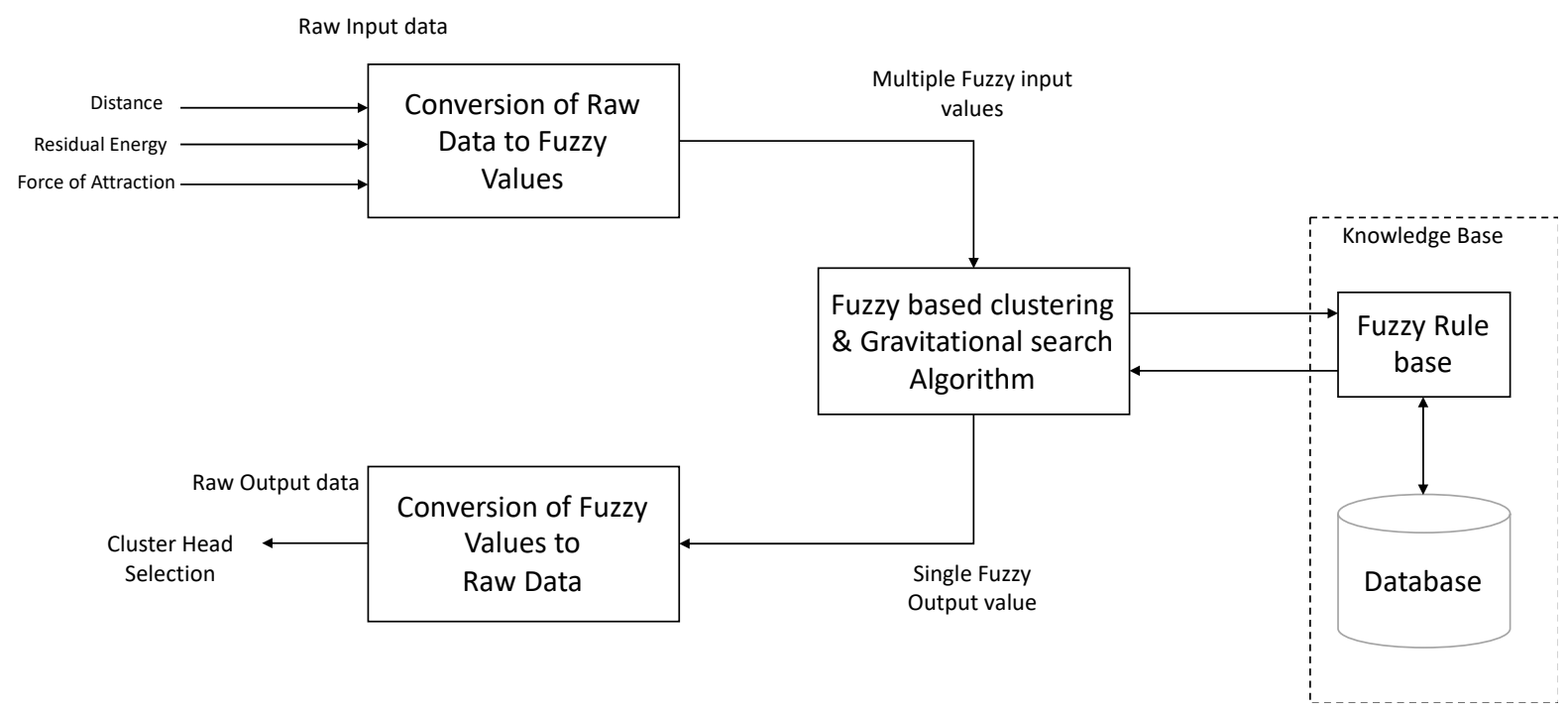

\section{Fig. 1. Block diagram of the proposed cluster head selection and routing model}

Rule based inference structure is provided using the linguistic knowledge with the help of fuzzy logic. In each cluster, the cluster head may be selected in an optimal manner with improved accuracy using this scheme. IF... THEN statements are used for representing the rules of the fuzzy inference system. Rule based manager, fuzzy inference, fuzzification and defuzzification phases are performed for obtaining the desired scalar variable as an 
output for the input fuzzy vector variable using fuzzy logic. Fuzzy inference system is used for selection of the cluster head. Distance, nodes residual energy and force of attraction are the three input variables in the raw data during fuzzification that are converted into higher, medium and low level linguistic variable representation. High, moderate and low level linguistic variables represent the force of attraction. High, adequate and low level linguistic variables represent the residual energy. Short, moderate and long linguistic variables represents the distance values. Membership values represent the input variables provided to the fuzzy inference system. Single fuzzy values are produced by combining multiple fuzzy input variable values. Distance, residual energy and force of attraction parameters are used for selection of the optimal cluster head in each cluster by combining the fuzzy values with the help of the fuzzy inference system. The databased and fuzzy rule base are available in the knowledge based in the proposed inference system. Fuzzy rules are stored by the rule base manager for obtaining the outputs. The input-output variable membership function is stored in the database. For selection of cluster head, conversion of fuzzy data into raw values is performed using defuzzification process by feeding the single and aggregated fuzzy output.

\section{Results and Discussion}

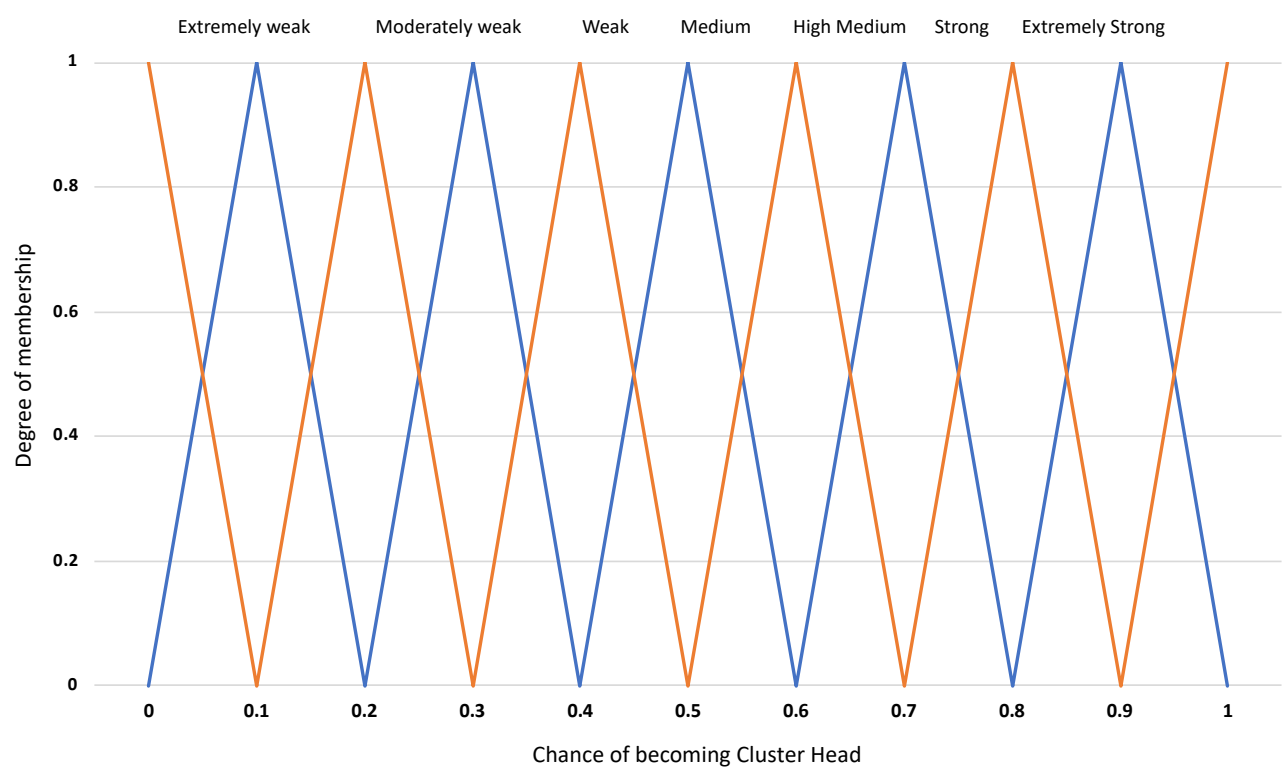

Fig. 2. Output set of cluster head selection analysis 
The linguistic value based analysis of output variables is performed. Extremely strong, strong, high medium, medium, weak, moderately weak and extremely weak membership function categories are available for selection of cluster head. Figure 2 represents the output set of the cluster head selection analysis. Membership functions with respect to distance, residual energy and force of attraction are used in the proposed fuzzygravitation based system. Mamdani fuzzy inference system (FIS) and seven membership function based cluster head selection probability is the output of the model. The routing process is enhanced for effective decision making using the FIS system. The higher, moderate and less value linguistic variables are used for demonstrating the force of attraction parameter using the fuzzy set membership function. Effective modelling of the residual energy levels can be done using this system. Short, moderate and long linguistic variables represent the input fuzzy set distance based membership function. The probability of selection of cluster head varies with the variation in the membership function and the input variable distances. Extremely strong, strong, high medium, medium, weak, moderately weak and extremely weak membership variables based on the final fuzzy output set affects the probability of selection of cluster heads. Figure 3 provides the accuracy analysis of the proposed model in comparison with the existing algorithms. It is evident that the proposed system is more efficient than the existing models in terms of accuracy.

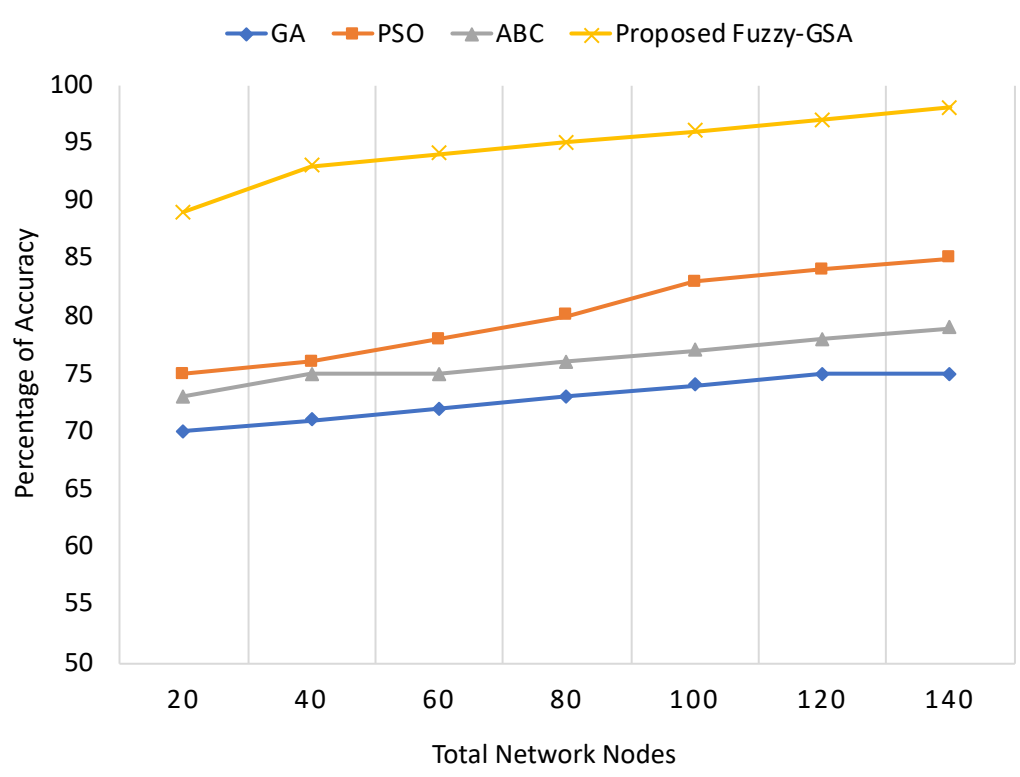

Fig. 3. Accuracy of clustering for the proposed algorithm 
MATLAB simulations are used for implementing the proposed routing model. Sensor nodes ranging between 20 to 1000 are used for performing the simulated experiments over a geographical region within 200 meters. $0.5 \mathrm{~J}$ is set as the initial value of very WSN node in this model. The simulation parameters involved in this simulation are data packet size, initial energy level, routing protocol, cluster head selection, clustering protocol, total clusters, total sensor nodes and area. Communication delay, energy consumption, network throughput, energy level of the node and network lifetime are analyzed using the proposed model. From the simulation results, it is evident that the proposed model offers better output when compared to the existing models. Fuzzy based, grid based, LEACH, gravitation based and the proposed algorithms are compared for the routing schemes as represented in figure 4 . Other techniques inclusive of fuzzy based cluster formation protocol (FBCFP), energy efficient grid based routing (EEGBR), fuzzy based unequal clustering (FBUC) and Fuzzy energy-aware unequal clustering (EAUCF) are also analyzed. The network lifetime is improved with the proposed fuzzy-gravitation based algorithm when compared to the existing algorithms. Several factors are responsible for this increase in lifetime. The clustering approach based on gravitational force improves the accuracy of clustering. Identification and categorization of all outliers into appropriate clusters is performed by checking and validating the clusters using fuzzy based rules. Sink node is reached by the data packets with specific number of hops that is reduced by the proposed unequal clustering model. Further, the clusters are refined after they are formed using a combined metric at the final stage.

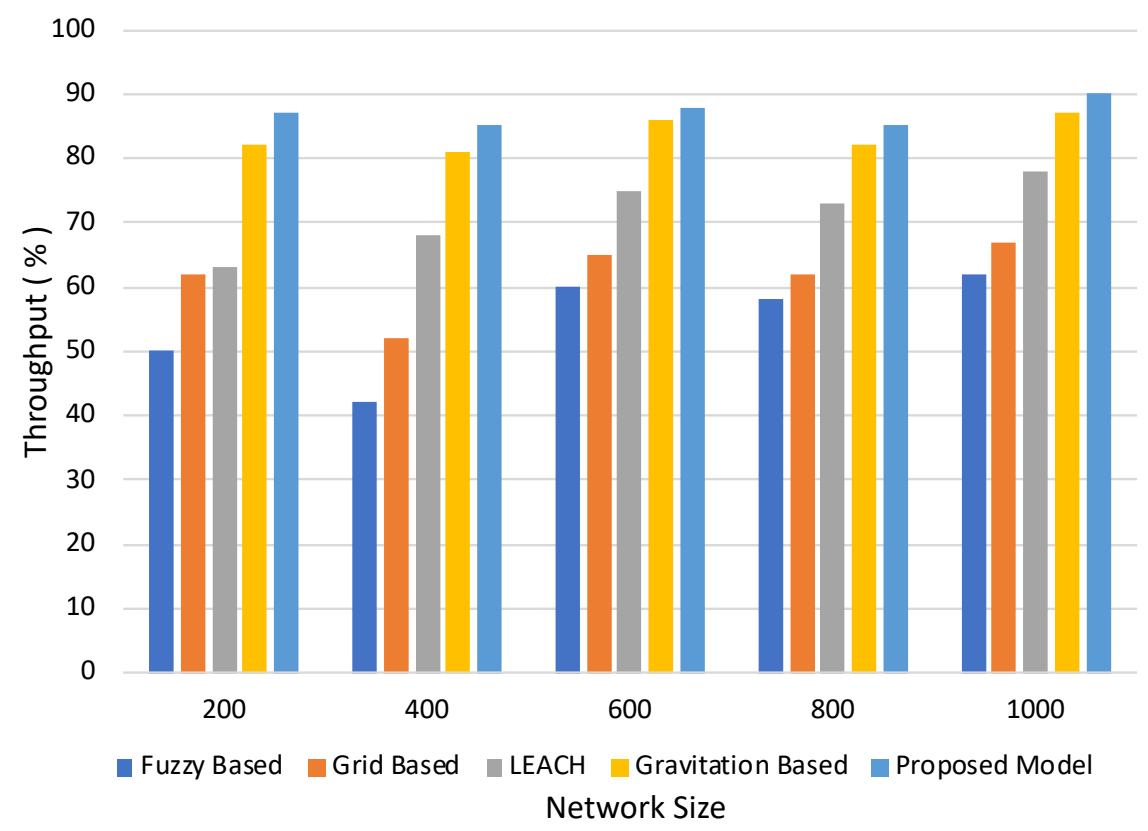

Fig. 4. Throughput analysis for the proposed model 


\section{Conclusion}

A fuzzy based routing and chaotic gravitational search algorithm is applied on a WSN for enhancing the network lifetime by improving the accuracy of clustering and enhancing the efficiency of the routing process. Effective decision making is done using fuzzy rules based on the clustering mechanism for forming perfect clusters and routing using the heuristic approach for data transmission through the most optimal routing path. This new routing scheme decreases the energy consumption and delay in data communication while increasing the WSN throughput, energy level of the participating nodes and the network lifetime. In this novel routing scheme, the overall performance is enhanced by a combination of metrics and fuzzy rules for selection of cluster heads. Increased network lifetime and decrease in energy utilization is ensured by comparing the proposed model with the conventional grid based models. Future work is directed towards application of the available renewable energy for enhancing the communication across nodes using intelligent agent based schemes.

\section{References}

[1] Zhou, S., Xu, X., Xu, Z., Chang, W., \& Xiao, Y. (2019). Fractional-order modeling and fuzzy clustering of improved artificial bee colony algorithms. IEEE Transactions on Industrial Informatics, 15(11), 5988-5998.

[2] Chen, J., Qi, X., Chen, L., Chen, F., \& Cheng, G. (2020). Quantum-inspired ant lion optimized hybrid k-means for cluster analysis and intrusion detection. KnowledgeBased Systems, 203, 106167.

[3] Rath, M., Pati, B., \& Pattanayak, B. K. (2018). Relevance of soft computing techniques in the significant management of wireless sensor networks. Soft Computing in Wireless Sensor Networks, 86-106.

[4] Shahidinejad, A., \& Barshandeh, S. (2020). Sink selection and clustering using fuzzy-based controller for wireless sensor networks. International Journal of Communication Systems, 33(15), e4557.

[5] Raj, J. S. (2019). QoS optimization of energy efficient routing in IoT wireless sensor networks. Journal of ISMAC, 1(01), 12-23. 
[6] Panag, T. S., \& Dhillon, J. S. (2021). Predator-prey optimization based clustering algorithm for wireless sensor networks. Neural Computing and Applications, 1-21.

[7] Lalwani, P., Banka, H., \& Kumar, C. (2017). GSA-CHSR: gravitational search algorithm for cluster head selection and routing in wireless sensor networks. In Applications of Soft Computing for the Web (pp. 225-252). Springer, Singapore.

[8] Kumar, M. M., \& Chaparala, A. (2019). OBC-WOA: opposition-based chaotic whale optimization algorithm for energy efficient clustering in wireless sensor network. intelligence, 250(1).

[9] Jannu, S., Dara, S., Kumar, K. K., \& Bandari, S. (2017, September). Efficient algorithms for hotspot problem in wireless sensor networks: gravitational search algorithm. In The International Symposium on Intelligent Systems Technologies and Applications (pp. 41-53). Springer, Cham.

[10] Pandey, A., Dey, A., \& Nandi, A. (2020). Lifetime Enhancement of Supernode Based WSNs with Optimal Size Cluster Formation by Using Gravitational Search Algorithm. In Proceedings of the 2nd International Conference on Communication, Devices and Computing (pp. 669-678). Springer, Singapore.

[11] Karunakaran, V. (2019). a stochastic development of cloud computing based task scheduling ALGORITHM. Journal of Soft Computing Paradigm (JSCP), 1(01), $41-48$

[12] Mugunthan, S. R. (2020). Novel Cluster Rotating and Routing Strategy for software defined Wireless Sensor Networks. Journal of ISMAC, 2(02), 140-146.

[13] Haoxiang, W., \& Smys, S. (2019). Qos enhanced routing protocols for vehicular network using soft computing technique. Journal of Soft Computing Paradigm (JSCP), 1(2), 91-102.

[14] Anand, J. V. (2020). Trust-Value Based Wireless Sensor Network Using Compressed Sensing. Journal of Electronics, 2(02), 88-95. 